\title{
Cheating with robots: how at ease do they make us feel?
}

\author{
Sofia Petisca ${ }^{1}$, Francisco Esteves ${ }^{2}$ and Ana Paiva ${ }^{3}$
}

\begin{abstract}
People are not perfect, and if given the chance, some will be dishonest with no regrets. Some people will cheat just a little to gain some advantage, and others will not do it at all. With the prospect of more human-robot interactions in the future, it will become very important to understand which kind of roles a robot can have in the regulation of cheating behavior. We investigated whether people will cheat while in the presence of a robot and to what extent this depends on the role the robot plays. We ran a study to test cheating behavior with a die task, and allocated people to one of the following conditions: 1) participants were alone in the room while doing the task; 2) with a robot with a vigilant role or 3) with a robot that had a supporting role in the task, accompanying and giving instructions. Our results showed that participants cheated significantly more than chance when they were alone or with the robot giving instructions. In contrast, cheating could not be proven when the robot presented a vigilant role. This study has implications for human-robot interaction and for the deployment of autonomous robots in sensitive roles in which people may be prone to dishonest behavior.
\end{abstract}

\section{INTRODUCTION}

Dishonesty is not a new problem. Research shows that cheating in educational settings is widespread [1], and different studies have already shown that if people have the opportunity and a minimum risk of being caught, as long as they can maintain a positive (honest) self-image of themselves, they will be dishonest in order to obtain some gain (e.g.[2], [3]). Hence, it is of utmost importance to create ways to inhibit the temptation of behaving dishonestly.

Apart from educating people, surveillance is without a doubt the most common way that societies use to prevent this kind of behavior. Vigilant security officers in shops or at events, cameras in the street, at work, or even at someone's home are common security measures. All these measures have one common characteristic: they remind us that someone might be watching, which has been shown to increase prosocial behavior[4].

As autonomous robots and machines become more pervasive in our environment, one can ask if they can play a role in influencing people towards more moral behaviors. Knowing

Sofia Petisca acknowledges an FCT grant (Ref.SFRH/BD/118013/2016)

${ }^{1}$ Sofia Petisca is with INESC-ID and Instituto Universitário de Lisboa (ISCTE-IUL),CIS, Portugal sofia.petisca@inesc-id.pt

${ }^{2}$ Francisco Esteves with Mid Sweden University, Sweden and Instituto Universitário de Lisboa (ISCTE-IUL)

${ }^{3}$ Ana Paiva with INESC-ID,Instituto Superior Tecnico, University of Lisbon, Portugal

IEEE/RSJ International Conference on Intelligent Robots and Systems (IROS), Macau, China, November 4-8, 2019 (C) 2019 IEEE. Personal use of this material is permitted. Permission from IEEE must be obtained for all other uses, in any current or future media, including reprinting/republishing this material for advertising or promotional purposes, creating new collective works, for resale or redistribution to servers or lists, or reuse of any copyrighted component of this work in other works that some jobs are more sensitive and prone to dishonesty from people, can a robot have those tasks? And how can we better equip robots to discourage dishonest behaviors? Two studies in Human-Robot Interaction (HRI) started to explore these questions, using a non-social robot with a vigilant role [5], [6]. But a gap still exists in the roles a robot can have when dishonesty is tempting. For example, although it is still at an early stage, in care settings, social robots are being developed to assist people (e.g.[7]). In these settings, dishonesty might arise in the form of not wanting to take the pill prescribed or not following the diet plan. Therefore, it is necessary to understand if a more social robot can prevent these dishonest acts.

With this purpose, we developed a study where we asked participants to play a die game in which they were promised a tempting monetary reward if they achieved a certain score. The design of the game allowed participants to cheat to obtain a higher score.

We randomly allocated participants to one of the following conditions: 1) alone in the room while doing the task (Alone Condition); 2) with a vigilant robot that did not speak (Vigilant Robot Condition) and 3) with a robot that watched and spoke (Robot Gives Instructions Condition).

We hypothesized that people in the Alone Condition would exhibit cheating behavior (Hypothesis 1). On the other hand, it was observed that having a human in the room [8] or a non-social robot [5] watching, inhibits the cheating behavior. So, we wanted to re-examine this effect with a robot watching more closely and directly tracking the participant. We hypothesized that, in this condition, the cheating behavior would be inhibited(Hypothesis 2). Lastly, and the novelty of our study was to test if an autonomous robot that also watched the participant as well as showing a simple (scripted) interaction during the task (like a supporting role), would be enough to inhibit cheating behavior. This was a more exploratory hypothesis, but knowing that adding more social cues can improve the level of persuasiveness of a robot[9] we hypothesized that by speaking and watching the participant, the watchful behavior would have a greater effect and would possibly inhibit cheating behavior (Hypothesis 3).

Overall, and considering previous studies, we expected that participants would cheat more when they were alone, than when they were with the robots (Hypothesis 4). Results confirmed the alone assumption, but the two robots elicited completely different behaviors: the vigilant robot inhibited and the speaking robot dis-inhibited cheating. 


\section{RELATED WORK}

Studies show that when people see others breaking the rules, they also tend to violate them, causing the spread of disorder [10]. Much like having a broken window and signs of abandonment can rapidly prod people to misbehave[11]. With dishonesty being so widely broadcasted every day, it is even more pressing to find ways to discourage it.

The fields of Psychology and Economics have extensively explored the factors that facilitate and inhibit cheating behaviors. It was thought that dishonest behavior emerged only from external rewards, from a cost-benefit analysis: the amount gained, the probability of being caught and the punishment if caught. Recently, it has been shown that internal rewards also play a role in the decision to be dishonest. Namely, whether the dishonest act alters our idea of being an honest person[2]. According to this, dishonest behavior is exhibited, as long as people can justify their actions while still perceiving themselves to be honest people (protecting their self-concept). This is in line with the Objective SelfAwareness theory, that shows us that by bringing selfawareness to the self there is a comparison with standards, when a discrepancy appears, there is a motivation to try and get to a consistent self [12] [13].

Examples of the effect of self-awareness can be seen in decreasing cheating behavior, when people have to see their own reflection and hear their own voice while doing a task[14], when they sign an honor code or read a moral reminder[2] or even just by increasing the time given to perform a task[15]. Making people aware of their actions, breaks the illusion of being honest and inhibits the dishonest act in order to get to a coherent self. Another way in which people are more commonly made self-aware is through surveillance. Studies done with humans, even show that people act in a socially desirable and prosocial way, when being watched [4]. With dishonesty, the power of being watched also decreases cheating behavior either with a close surveillance, like having someone watching participants doing a task[8] or just by guaranteeing participants that all answers will be checked[16].

It is plausible to assume that robots can, in the future, perform a various range of tasks useful for society[17]. They can assist in more mechanical and dangerous tasks (e.g.[18]) or they can assist as social robots working alongside with humans (e.g.[19], [20]). Furthermore, machines are seen as social actors, and it seems that people treat them just like other humans[21] opening a window for the use of machines as persuasive technology. Robots also seem to have a persuasive effect on humans, persuading them to consume less energy[22] or by persuading people on the aversive consequences of lying[9].

Studies on human dishonesty in HRI are still scarce, but a first study already started to explore this, finding that people cheated more when they were alone in a room than when they were with a researcher or a robot that just did random eye gaze, suggesting that the robot as it was, inhibited in the same amount as the human watcher [5].
Contrary to this, another study ran in a natural setting showed that people stole more snacks when a table was left unattended, or when a robot watching was present as opposed to when a human was monitoring it. As the authors explain, people may have felt that the robot was not able at all to judge them and catch them in their dishonesty[6]. In addition, the fact that people were with others might have had an effect that distinguishes the different results of these two studies.

Overall, these first studies were important steps to understand the behavior of people around robots when honesty is at stake.

Therefore, knowing that social robots are being prepared to be able to live alongside humans, e.g. helping at home with medication or improving their health, it is important to understand if a more interactive robot can also inhibit dishonest actions. With this in mind, the novelty of our research is trying to understand if, by giving more capabilities to our watchful robot, like accompanying someone in a simple way while doing a task, if it still inhibits a dishonest act.

\section{METHOD}

We designed a study to test if people would be dishonest with robots, manipulating the role they had in the task. The robot would either simply have a vigilant role, like a surveillance camera, or it would show more interaction (speaking), with a more supporting role, accompanying the participant during the task. Our control condition was being alone in the room.

\section{A. Sample}

We recruited 76 participants from a Portuguese university, students and researchers, of which four were excluded because they were younger than 18 years. This resulted in a sample of 72 participants with 50 males and 22 females, with ages ranging from 18 to 48 years $(M=22.63, S D=4.960)$. All participants signed a consent form and were randomly assigned to one of the conditions. We used EMYS robotic head[23] with a Kinect version 2 for tracking participants position (for a more natural gaze behavior) and the SERA tools[24]. The die task was done on a laptop, the questionnaires were answered in paper at a separate table and the sessions took approximately 30 minutes. The room where the sessions took place had no furniture besides the tables and chairs needed for the tasks, so that participants could know that no camera was hidden, and anonymity was assured.

\section{B. Die Task}

The task used to ascertain the cheating behavior was done with a virtual die (adapted from [25]). Participants had to throw the die 20 times and for each throw they had to guess where the highest number would appear: on the top or the downside of the die. Each side they reported would earn them points (they had Table 1 for help). For example, if they chose the downside and it was a 5 on top, they would win 2 points.

They were told that if they made 75 or more points, they would win 5 euros. Participants were asked to follow these 
TABLE I

DIE NUMBERS IN THE CORRESPONDING SIDES:

\begin{tabular}{|l|l|l|l|l|l|l|}
\hline Up & 1 & 2 & 3 & 4 & 5 & 6 \\
\hline Down & 6 & 5 & 4 & 3 & 2 & 1 \\
\hline
\end{tabular}

rules:

1st) Choose for yourself which side you think the highest number will appear (up or down).

2nd) Throw the die.

3rd) Report which side you had previously guessed.

Since the choice was done in their minds and only reported after seeing the outcome, there was an opportunity to cheat without proof.

Normally, these kinds of studies are done with a random die, but we opted by using an unfair die, because this way we had exactly the same amount of type of numbers for each participant. Moreover, to create a way to persuade participants to be dishonest, we had more 1's and 6's than other numbers. The sequence for the 20 throws was as follows:

$$
\text { “3, 6, 1, 4, 2, 1, 5, 6, 1, 2, 4, 5, 1, 3, 2, 6, 2, 1, 1, 6" }
$$

The program would save each number presented and the respective participant choice. The 75 points were decided as a cutting point for giving the reward, but the cheating behavior was inferred through the probability of each number choice (see results section).

\section{Cautions taken into account for studying cheating behav- ior}

Cheating behavior is not an easy behavior to study, first because people who cheat do not normally do it to the full extent, but just a little (e.g.[2], [26], [27]) and just by feeling watched people refrain from the behavior. For these reasons we were very careful in creating an anonymous environment to run the sessions. First, for data logging, we assigned participants a participant number that did not identify them in any way. Second, we did not debriefed participants after the session. Typically, experimental studies that do not fully disclose the study objective prior to the experiment, are concluded with a debriefing to inform participants about the objective of the study. However, studies on dishonest behavior do not usually apply a debriefing, since it can be very harmful for the participant well-being (e.g.[28], [2], [29], especially for people who had in fact cheated to get the reward.

Therefore, we offered the participants with the possibility to request more information about the study by leaving their e-mail address in a sheet of paper left on the table where the questionnaires were answered.

This way, a general email could be sent to everyone who signed it stating the true purpose of the study and the general results from the sample studied, minimizing the risk in the moment of participants feeling discomfort if they cheated.

\section{Study Conditions}

Participants were randomly allocated to one of these conditions:

1) Alone Condition (21 participants)- participants did the task all by themselves in the room. This condition was the control condition for cheating, in which we did not inhibit the participants' cheating behavior in any way.

2) Vigilant Robot Condition (26 participants)- Emys was right next to the participant in the table to convey the role of a vigilant robot and was capturing the participant position, looking directly at him/her for the duration of the task. Emys never interacted verbally with the participant and no information was given to why the robot was there. This condition served to re-test the effect of the robot found in a previous study[5].

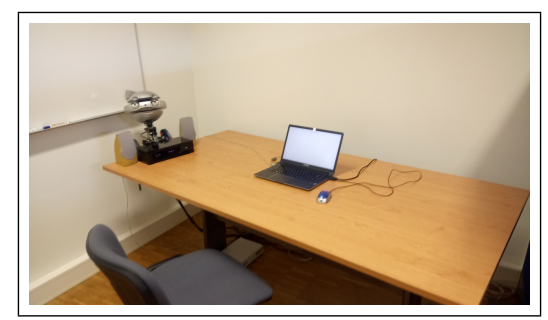

Fig. 1. Vigilant Robot Condition- where Emys just looked at the participant with face tracking behavior and no other kind of interaction.

3) Robot Gives Instructions Condition (25 participants)Emys would be in front of the participant giving the instructions for the die task and accompanying them until the end of the game, while also showing the same natural gaze behavior of tracking the participant as in the other robot condition. This condition served to test if a more interactive and watchful robot would influence cheating. Therefore, after giving the instructions the robot would warn when ten throws were left, and at the end it would provide the total collected points and say goodbye (this interaction was following a script).

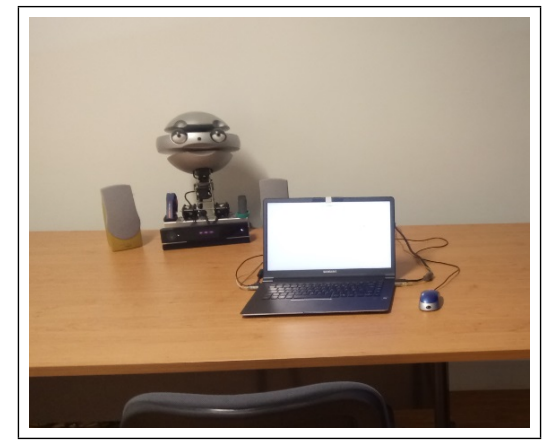

Fig. 2. Robot Gives Instructions Condition- Emys was giving the instructions of the task and accompanying the participant while doing face tracking behavior. 


\section{E. Procedure}

To elicit people's natural behavior and explore their cheating behavior, we told participants that the goal of the study was to understand people's capabilities of predicting the future when there was a reward, compared to when there was not.

After arriving at the room, participants had to read and sign the consent form of the study, which explained the cover story, guaranteed that all data from the participants was anonymous and the session would not be video, or audio recorded. Then, participants were randomly assigned to only one of the conditions and started by doing two filling tasks (guessing a color they would take out of an envelope and a word hunt task) in order to not draw much attention to the die task in which we were measuring their cheating behavior.

Next, they moved to another table where they did the die task in a computer with a virtual die and if they made 75 or more points, they would win 5 euros. In the Alone Condition participants did the task with no one else in the room, having a paper next to the computer with the instructions.

In the Robot Gives Instructions Condition the robot was already tracking the participant when he approached the table and the script started as soon as participants clicked "Start" on the screen. In the Vigilant Robot Condition, nothing was told about the robot, participants just saw it by approaching the table and it was already looking directly at them. A paper next to the computer had all the instructions for the task.

Upon finishing it, participants moved to another table (where they could not see the robot) and answered a questionnaire. When finished, they were asked by the researcher if they had made 75 or more points, if so, they would receive 5 euros (approximately 5.8\$ USD at the time of collection) and be thanked for their participation.

\section{F. Measures}

Demographic information (age, gender) was requested along with some cover-story questions (these were not analyzed, since they were just there to mask the objective of the study).

Then, participants filled the HEXACO-60 Personality Inventory [30] (we used an adapted and validated version for Portuguese population[31]). This Inventory assesses the six dimensions of the HEXACO model of personality structure, with 10 items for each of the dimensions: Honesty-Humility; Emotionality; Extraversion; Agreeableness; Conscientiousness and Openness to Experience. It has some items that need to be reversed and then an average is taken for each dimension.

We only analyzed the Honesty-Humility dimension, which we wanted to see if it had any relationship with the cheating behavior. This questionnaire has 60 items with a 5-point Likert scale ranging from 1-Totally Disagree to 5-Totally Agree.

After, in 5-point Likert scale participants reported how much they feel watched or watched by the robot, ranging from 1- Totally Disagree to 5- Totally Agree.
Regarding cheating behavior, we used an unfair die, but since the participant did not know that and they had to make 20 throws, we assumed that their choices would be random and probability of success (choosing the higher outcome) being 0.50 . By comparing the average probability of success of each condition with 0.50 we could see if participants were getting a significantly higher amount of success than randomand thus, infer cheating. Participants would only report the side they had chosen after seeing the die outcome, so they could change this choice to be more favorable to them.

\section{RESUlTS}

The literature is mixed regarding differences between gender in cheating (e.g.[27], [32], [33], [34]) and to control for this we checked if any gender differences existed regarding the probability of successes reported in each condition, no significant differences were found so we did not include this factor in the following analyzes.

Our primary objective was to see if people cheated in the different conditions, for this we calculated the probability of guessing the highest number in 20 throws for each participant. Participants could either get in a throw success (guessing the highest), or failure (guessing the lower), we gave a value of 1 to a success and a value of zero to a failure. And with this we calculated the probability of success, by adding the number of successes per participant and then dividing by the 20 throws.

Only one participant (instructions condition) fully cheated, choosing the best outcome for each throw. This supports the notion that normally people do not cheat to the maximum extent. Besides this, only 4 participants distributed across the conditions always chose the same side throughout the 20 throws (the up side), but since their success probability was under 0.5 we did not exclude them from the analyses.

The averages of the probability of success per condition were: Alone condition $(M=.59, S D=.120)$; Vigilant Robot $(M=.53, S D=.131)$ and Robot Giving the Instructions $(M=.58, S D=.164)($ see Fig. 3$)$.

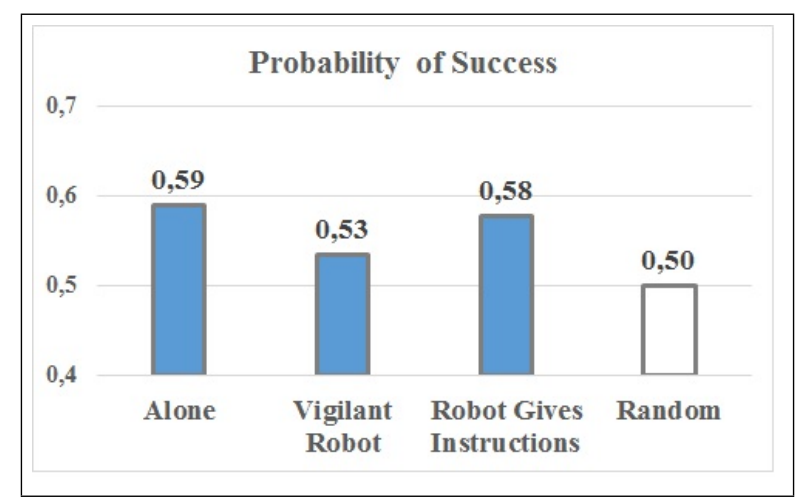

Fig. 3. Averages of Probability of Success per condition, compared to chance.

After checking for normality with the Shapiro-Wilk test, we used the One-sample T-test to check for differences between the success probability in each condition and the random probability of success of 0.5 . 
Alone Condition $(t(20)=3.455, p=.003)$, in this condition as expected there was a significant difference, i.e. cheating behavior could be inferred in this group.

Vigilant Robot Condition $(t(25)=1.340, p=.192)$, for this condition, there was no significant differences from chance, i.e. cheating behavior could not be observed in this group.

Robot Gives Instructions Condition $(t(24)=2.369$, $p=.026)$, in this condition, there was a significant difference from chance, i.e. cheating behavior was observed.

We then analyzed if there were any differences between the conditions. For this, we ran a One-way ANOVA with the probabilities of success and we did not find any significant differences $(F(2,69)=1.051, p=.355)$.

Regarding the Honesty-Humility Dimension we found a significant negative correlation between the probability of success in the Robot Gives Instructions Condition and the Honesty score $(\mathrm{r}=-.447, \mathrm{p}=.025)$, suggesting that in that condition when the rate of success was higher the Honesty score was lower and vice-versa.

Regarding participants' perceptions of being watched (5point Likert scale) they reported feeling watched in the Vigilant Robot $(M=3.62, S D=.278)$ and the Robot giving the instructions $(M=3.88, S D=.247)$, although there was no statistically significant difference between these scores $(U=293.5, p=.534)$, they felt equally observed by both robots. In the Alone condition they did not feel watched at all $(M=1.4)$.

\section{DISCUSSION}

Cheating is a complex behavior, and some people will do it if they have something to gain and minimum risk of being caught. We used a task in which participants could anonymously cheat to get a higher score without revealing their choice to others.

When participants were alone in the room, they presented a significantly higher success rate than chance (supporting our Hypothesis 1), which was expected, since no one was watching. There was no kind of reminder that they were doing something dishonest and the risk of getting caught was null.

Knowing that having someone watching inhibits cheating, we then explored two different roles that robots could have. We had a vigilant robot, to replicate the effect obtained in[5] with a robot scanning a room from a distance. We found that success rate was not significantly different from chance, which shows that the passively observing robot inhibited cheating behavior in participants (supporting our Hypothesis 2). However, when we used a watchful robot with a more interactive and supporting role, the results shifted. Participants cheated significantly more than chance, disconfirming our Hypothesis 3.

These results seem to suggest that with the vigilant robot, participants might have felt more suspicious and insecure, they did not know what the robot was doing, and thus, this might have contributed to less cheating. Similar to the feeling of being observed by a security camera, where we do not know if someone is watching. In contrast, when the robot was giving the instructions, the inhibiting effect was lost. In both conditions, the robots gaze behavior was equally watchful, leaving us with the only difference that differentiated both robots- one accompanied the task verbally and the other did not. It could be that the instructions robot made it more obvious the extent of its capabilities, that it could not catch the participant in a lie. Thus, acknowledging the robot capabilities may have surpassed the inhibiting effect of the watching behavior on the condition where the robot was verbally interacting with the participant. Participants may have felt more at ease opening more space to justify their dishonest acts. These results suggest that in more complex environments where a social robot is needed, the robot capabilities need to be carefully considered and its limitations should not be easily perceived.

Regarding our last hypothesis, we did not find a significant difference between the conditions, which may be due to a small sample size (less than 27 subjects per condition) and cheating being a small effect, so we cannot say they cheated more in one than the other (not confirming our Hypothesis 4). Nevertheless, there was cheating behavior present in some conditions, while in others this effect was not different from chance.

Regarding the Honesty-Humility dimension, we only found a significant negative correlation with cheating for the Robot Giving Instructions Condition. Suggesting, that when cheating was higher in this condition, participants presented a lower level of Honesty-Humility. For the other conditions this correlation was not significant.

\section{LimitaTiONS}

There are some limitations to the study that need to be addressed. First, the robots in the two robot conditions were placed in different locations on the table. Since we wanted one of the robots to be very vigilant, we put it in a position where it could see the computer screen. While the other robot was facing the participant while giving the instructions. But for a clearer design the robots should have been put in the same position. However, we do not believe their positions influenced the cheating behavior, because there were no significant differences between the scores given to both robots regarding feeling watched by it (and participants reported feeling very watched by the robots) and in a previous study[5] there was no access to the screen and the robot still inhibited cheating behavior. However, in future studies the robot position should be the same.

Additionally, we think that using an unfair die did not bring us more advantage than using a random one so in future studies we should change this approach. Furthermore, the participant's perceptions of the robots and their social presence were not explored but will be in future studies, since these could bring insightful data to accompany our results. 


\section{CONCLUSION}

In conclusion, since robots in the future could have roles where dishonesty might be tempting, it is important to see if they are able to have those roles.

By comparing the two robot conditions with the alone condition we could not find significant differences between them, suggesting that none was more effective than the other in preventing cheating behavior. But this might be because we had a small sample for this kind of behavior and high individual differences. In future studies this should be addressed in order to better understand the different dynamics in cheating behavior. Still, we were able to verify the level of cheating behavior in each condition. This way, we were able to ascertain if, in general, people were cheating or not.

The results showed that being with a more vigilant and "unknown" robot inhibited cheating behavior. However, the novelty of this study is that when tempted for dishonesty, interacting with a more interactive, but scripted robot seems to have the opposite effect. This has strong implications for the roles robots can have in the future, for example in people's homes, if they show limited capabilities, they might give space for people to be dishonest (e.g. lying on the daily pill intake). It will be interesting in future studies to explore if giving more intelligent capabilities to the robot will alter its effect, because in some situations there might be a need for a more social robot instead of just a vigilant one.

\section{ACKNOWLEDGMENT}

The authors thank the help and support of Filipa Correia when preparing this study and Sanne van Waveren in reviewing the manuscript. This work was supported by national funds through Fundação para a Ciência e a Tecnologia (FCT) with reference UID/CEC/50021/2019 and Sofia Petisca acknowledges an FCT Grant (Ref.SFRH/BD/118013/2016).

\section{REFERENCES}

[1] S. D. Blum, My word!: Plagiarism and college culture. Cornell University Press, 2009.

[2] N. Mazar, O. Amir, and D. Ariely, "The dishonesty of honest people: A theory of self-concept maintenance," Journal of marketing research, vol. 45, no. 6, pp. 633-644, 2008.

[3] D. Ariely and S. Jones, The (honest) truth about dishonesty: How we lie to everyone-especially ourselves. HarperCollins New York, NY, 2012, vol. 336.

[4] T. J. Van Rompay, D. J. Vonk, and M. L. Fransen, "The eye of the camera: Effects of security cameras on prosocial behavior," Environment and Behavior, vol. 41, no. 1, pp. 60-74, 2009.

[5] G. Hoffman, J. Forlizzi, S. Ayal, A. Steinfeld, J. Antanitis, G. Hochman, E. Hochendoner, and J. Finkenaur, "Robot presence and human honesty: Experimental evidence," in Proceedings of the Tenth Annual ACM/IEEE International Conference on Human-Robot Interaction. ACM, 2015, pp. 181-188.

[6] J. Forlizzi, T. Saensuksopa, N. Salaets, M. Shomin, T. Mericli, and G. Hoffman, "Let's be honest: A controlled field study of ethical behavior in the presence of a robot," in Robot and Human Interactive Communication (RO-MAN), 2016 25th IEEE International Symposium on. IEEE, 2016, pp. 769-774.

[7] R. Kachouie, S. Sedighadeli, R. Khosla, and M.-T. Chu, "Socially assistive robots in elderly care: a mixed-method systematic literature review," International Journal of Human-Computer Interaction, vol. 30, no. 5, pp. 369-393, 2014.
[8] M. K. Covey, S. Saladin, and P. J. Killen, "Self-monitoring, surveillance, and incentive effects on cheating," The Journal of Social Psychology, vol. 129, no. 5, pp. 673-679, 1989.

[9] J. Ham, R. Bokhorst, R. Cuijpers, D. van der Pol, and J.-J. Cabibihan, "Making robots persuasive: the influence of combining persuasive strategies (gazing and gestures) by a storytelling robot on its persuasive power," in International conference on social robotics. Springer, 2011, pp. 71-83.

[10] K. Keizer, S. Lindenberg, and L. Steg, "The spreading of disorder," Science, vol. 322, no. 5908, pp. 1681-1685, 2008.

[11] J. Q. Wilson and G. L. Kelling, "Broken windows," Atlantic monthly, vol. 249 , no. 3, pp. 29-38, 1982.

[12] S. Duval and R. A. Wicklund, "A theory of objective self awareness." 1972.

[13] P. J. Silvia and T. S. Duval, "Objective self-awareness theory: Recent progress and enduring problems," Personality and Social Psychology Review, vol. 5, no. 3, pp. 230-241, 2001.

[14] E. Diener and M. Wallbom, "Effects of self-awareness on antinormative behavior," Journal of Research in Personality, vol. 10, no. 1, pp. 107-111, 1976.

[15] S. Shalvi, O. Eldar, and Y. Bereby-Meyer, "Honesty requires time (and lack of justifications)," Psychological science, vol. 23, no. 10, pp. 1264-1270, 2012.

[16] I. Békir, S. E. Harbi, G. Grolleau, N. Mzoughi, and A. Sutan, "The impact of monitoring and sanctions on cheating: Experimental evidence from tunisia," Managerial and Decision Economics, vol. 37, no. 7, pp. 461-473, 2016.

[17] K. Dautenhahn, "Roles and functions of robots in human society: implications from research in autism therapy," Robotica, vol. 21, no. 4, pp. 443-452, 2003.

[18] R. R. Murphy, "Human-robot interaction in rescue robotics," IEEE Transactions on Systems, Man, and Cybernetics, Part C: Applications and Reviews, vol. 34, no. 2, pp. 138-153, 2004.

[19] B. Robins, K. Dautenhahn, R. Te Boekhorst, and A. Billard, "Robotic assistants in therapy and education of children with autism: can a small humanoid robot help encourage social interaction skills?" Universal Access in the Information Society, vol. 4, no. 2, pp. 105-120, 2005.

[20] K. Wada and T. Shibata, "Social effects of robot therapy in a care house-change of social network of the residents for one year-," Journal of advanced computational intelligence and intelligent informatics, vol. 13, no. 4, pp. 386-392, 2009.

[21] C. Nass and Y. Moon, "Machines and mindlessness: Social responses to computers," Journal of social issues, vol. 56, no. 1, pp. 81-103, 2000.

[22] C. Midden and J. Ham, "The illusion of agency: the influence of the agency of an artificial agent on its persuasive power," in International Conference on Persuasive Technology. Springer, 2012, pp. 90-99.

[23] J. Kędzierski, R. Muszyński, C. Zoll, A. Oleksy, and M. Frontkiewicz, "Emys - emotive head of a social robot," International Journal of Social Robotics, vol. 5, no. 2, pp. 237-249, 2013.

[24] T. Ribeiro, A. Pereira, E. Di Tullio, and A. Paiva, "The sera ecosystem: Socially expressive robotics architecture for autonomous human-robot interaction," in 2016 AAAI Spring Symposium Series, 2016.

[25] T. Jiang, "The mind game: Invisible cheating and inferable intentions," 2012.

[26] S. Shalvi, J. Dana, M. J. Handgraaf, and C. K. De Dreu, "Justified ethicality: Observing desired counterfactuals modifies ethical perceptions and behavior," Organizational Behavior and Human Decision Processes, vol. 115, no. 2, pp. 181-190, 2011.

[27] J. Abeler, A. Becker, and A. Falk, "Representative evidence on lying costs," Journal of Public Economics, vol. 113, pp. 96-104, 2014.

[28] D. M. Bersoff, "Why good people sometimes do bad things: Motivated reasoning and unethical behavior," in The next phase of business ethics: Integrating psychology and ethics. Emerald Group Publishing Limited, 2001, pp. 239-262.

[29] C.-B. Zhong, V. K. Bohns, and F. Gino, "Good lamps are the best police: Darkness increases dishonesty and self-interested behavior," Psychological science, vol. 21, no. 3, pp. 311-314, 2010.

[30] M. C. Ashton and K. Lee, "The hexaco-60: A short measure of the major dimensions of personality," Journal of personality assessment, vol. 91, no. 4, pp. 340-345, 2009.

[31] A. MARTINS, "Depressiva persistente," Ph.D. dissertation, Universidade de Aveiro, 2015.

[32] J. Childs, "Personal characteristics and lying: An experimental investigation," Economics Letters, vol. 121, no. 3, pp. 425-427, 2013. 
[33] Y. Arbel, R. Bar-El, E. Siniver, and Y. Tobol, "Roll a die and tell a liewhat affects honesty?" Journal of Economic Behavior \& Organization, vol. 107, pp. 153-172, 2014.

[34] A. Dreber and M. Johannesson, "Gender differences in deception," Economics Letters, vol. 99, no. 1, pp. 197-199, 2008. 